\title{
15
}

\section{Электротоковая тепловая дефектоскопия металлических пластин}

\author{
() Ю.И. Головин, А.И. Тюрин, Д.Ю. Головин, А.А. Самодуров
}

НИИ „Нанотехнологии и наноматериалы“ Тамбовского государственного университета им. Г.Р. Державина

E-mail: golovin@tsu.tmb.ru

\section{Поступило в Редакцию 10 марта 2017 г.}

Предложен и экспериментально апробирован электротермический способ неразрушающего контроля электропроводящих материалов. Он основан на видеорегистрации ИК-излучения поверхности, возникающего при возбуждении пластинчатого образца миллисекундным импульсом тока. Последующая компьютерная обработка картин нестационарного теплового поля позволяет обнаруживать дефекты миллиметрового и субмиллиметрового размера, а также оценивать их геометрические характеристики и коэффициент температуропроводности материала.

DOI: $10.21883 /$ PJTF.2017.19.45083.16769

Среди множества методов неразрушающего контроля и тепловой дефектоскопии металлических изделий, описанных, например, в [1-6], особый интерес представляют методы высокоскоростного кинофильмирования нестационарной тепловой картины в инфракрасном (ИК) диапазоне, индуцируемой пропусканием электрического тока [7-9]. Ток вызывает локальный разогрев объекта в области неоднородности. Такой подход требует использования источников тока большой импульсной мощности и дорогостоящих высокоскоростных тепловизионных камер. Существенно понизить требования к аппаратуре и сделать ее портативной могут адекватная компьютерная обработка теплового изображения и восстановление распределения внутренних источников тепла, обусловленных наличием тех или иных дефектов в материале/изделии. Цель настоящей работы состоит в определении положения, формы и геометрических размеров дефектов в металлических пластинах методом коррекции (восстановления) положения локальных джоулевых источников тепла с помощью оригинального программного обеспечения. 
Листовой образец стали 65 толщиной $1 \mathrm{~mm}$ нагревался импульсом тока с регулируемыми амплитудой $(30-150 \mathrm{~A})$ и длительностью (5-3000 ms). ИК-изображение кинофильмировали тепловизионной системой FLIR A35sc на базе неохлаждаемых $\mathrm{VO}_{x}$-микроболометров, обладающих наибольшей чувствительностью в диапазоне длин волн $\lambda=7.5-13 \mu \mathrm{m}$. Камера имела матрицу $320 \times 256$ пикселей с шагом датчика детектора $25 \mu \mathrm{m}$, максимальное угловое разрешение (IFOV) $2.78 \mathrm{mrad}$, чувствительность $\sim 0.05^{\circ} \mathrm{C}$ (в диапазоне температур от -20 до $+550^{\circ} \mathrm{C}$ ) и частоту выводимых и сохраняемых кадров $60 \mathrm{~Hz}$. Описанная система позволяла получить информацию о тепловых свойствах материала, наличии и размерах дефектов (как в исходном состоянии, так и после провоцирующего развитие дефектов локального нагружения [10]).

Для теоретической оценки распределения мощности источников тепловыделения в двумерной задаче использовалась теория конформных отображений дефектов в комплексной плоскости $z$ [11]. В случае круглого отверстия в бесконечной плоскости отображение Жуковского в виде $z+r^{2} / z$ (центр системы координат находится в центре отверстия, действительная ось ориентирована вдоль направления тока на бесконечности $j_{\infty}, r$ - радиус отверстия) позволило найти искомое распределение плотности тока. Плотность источников тепловыделения $q \sim j^{2}$, где $j-$ локальная плотность тока. Последняя пропорциональна модулю локального значения производной функции отображения, отсюда следует $q \sim\left|1-r^{2} / z^{2}\right|^{2}$. Таким образом, симметричные максимумы плотности тепловыделения $q_{\max }=4 q_{\infty}$ достигаются на границах отверстия в точках, лежащих на перпендикуляре к направлению $j_{\infty}$ $\left(q_{\infty}\right.$ - плотность тепловыделения на бесконечности). Распределение $q$ вдоль оси $y$, перпендикулярной $j_{\infty}$, описывается формулой $\left(1+r^{2} / y^{2}\right)^{2}$. На границе отверстия $q \sim 2(1-\cos 2 \varphi)$, где $\varphi-$ текущий угол относительно $j_{\infty}$.

Для теоретической оценки распределения $q$ в случае прямой трещины длиной $2 h$, ориентированной перпендикулярно вектору $j_{\infty}$, может быть использовано конформное отображение $\left(z^{2}+h^{2}\right)^{1 / 2}$ (центр системы координат находится в центре разреза, действительная ось направлена вдоль $\left.j_{\infty}\right)$. Аналогично случаю с круглым отверстием получаем $q \sim\left|z^{2} /\left(z^{2}+h^{2}\right)\right|$, что дает в окрестности кончика разреза расходимость вида $h / 2 r$, где $r-$ расстояние от вершины разреза. Грубо оценить регистрируемое превышение роста температуры над ростом температуры фона можно, усреднив $q$ в круге заданного радиуса с

5 Письма в ЖТФ, 2017, том 43, вып. 19 
центром в вершине разреза и подставив в качестве радиуса характерное расстояние тепловой диффузии $r_{0}=(\chi t)^{1 / 2}$, где $\chi-$ коэффициент температуропроводности материала, $t$ - время с момента начала импульса тока. Если $r_{0} \ll h$, то такая процедура дает отношение максимальной температуры к температуре на бесконечности $h /(\chi t)^{1 / 2}$. Таким образом, увеличение длительности импульса тока приводит к соответствующему понижению контрастности термограммы, регистрируемой в конце импульса.

С другой стороны, использование коротких импульсов тока лимитируется множеством факторов, следующих, в частности, из необходимости более высоких значений $j_{\infty}$ для создания достаточных локальных перегревов объекта по сравнению с шумом на термограмме. Создание высоких $j_{\infty}$ требует мощных источников тока и вызывает сильные импульсные электромагнитные помехи, влияющие на работу всей аппаратуры. Кроме того, короткие импульсы требуют большего быстродействия и пространственного разрешения регистратора ИК-изображения, большей точности синхронизации с моментом регистрации термограммы и др. Каждый из этих факторов приводит к существенному увеличению стоимости установки.

Таким образом, с практической точки зрения представляет интерес разработка методик обнаружения и определения длины трещин, использующих относительно длинные импульсы тока невысокой плотности, что особенно существенно для изделий большого сечения. Уменьшение контрастности термограммы и соответственно увеличение влияния шумов должны компенсироваться совершенствованием алгоритмов анализа термограмм. Для построения таких алгоритмов использовалось компьютерное моделирование процессов распространения тепла.

Численное моделирование тепловой задачи проводилось в приближении заранее известного распределения мощности тепловых источников, полученного из аналитического решения задачи протекания тока по бесконечной пластине с заданным дефектом (круглым либо тонким прямолинейным). Далее решалось неоднородное уравнение теплопроводности с постоянными коэффициентами и адиабатическими граничными условиями с использованием явной схемы вычислений на равномерной квадратной сетке с пространственным шагом, соответствующим разрешению ИК-камеры в каждом конкретном случае (обычно 50-100 $\mathrm{m}$ ), и временны́м шагом $10 \mu \mathrm{s}$, что обеспечивало сходимость схемы для значений $\chi$ вплоть до $10^{-3} \mathrm{~m}^{2} / \mathrm{s}$ (табличные 
значения $\chi$ для стали $\left.12 \cdot 10^{-6}-15 \cdot 10^{-6} \mathrm{~m}^{2} / \mathrm{s}\right)$. Теплоотводом с поверхности пластины пренебрегалось, так как при коэффициенте теплоотдачи порядка $10 \mathrm{~W} /\left(\mathrm{m}^{2} \cdot \mathrm{K}\right)$ и перегреве в несколько градусов относительное изменение температуры за одну секунду не превышает $1 \%$. Ввиду недостаточности информации об электропроводности образца и излучательной способности его поверхности значения температур, полученные в результате моделирования, масштабировались для наилучшего соответствия результатам эксперимента.

На рис. 1, $а$ показана экспериментально полученная разность тепловых полей непосредственно до и после прохождения прямоугольного импульса тока по стальной пластине с круглым отверстием. На рис. 2, a приведены профили температуры, полученные экспериментально и по результатам моделирования для разных моментов времени вдоль оси $y$, перпендикулярной $j_{\infty}$. Хорошее согласие результатов моделирования и эксперимента позволяет использовать круглое отверстие в качестве просто создаваемого и хорошо определенного модельного объекта для нахождения коэффициента температуропроводности исследуемого материала и калибровки аппаратуры. При наличии отверстия известного диаметра этот метод позволяет определять $\chi$ с точностью лучше чем $10 \%$.

На рис. $1, b$ показана разность тепловых полей непосредственно до и после прохождения прямоугольного импульса тока длительностью $733 \mathrm{~ms}$ по стальной пластине с боковой перпендикулярной трещиной с длиной $6.4 \mathrm{~mm}$ и раскрытием 50-100 $\mu \mathrm{m}$, вводимой методом индентирования [10]. На рис. 2, $b$ профили температуры вдоль оси $y$, перпендикулярной $j_{\infty}$, представлены для разных моментов времени $t$ от переднего фронта импульса тока. Хорошее согласие результатов моделирования с экспериментом позволяет использовать эти профили для оптимального выбора функций, которые применяются для аппроксимации тепловых полей, создаваемых при обтекании током трещин неизвестной длины, с целью наиболее точного определения положения ее вершины по сильно зашумленной термограмме в автоматическом режиме.

Для острых и протяженных дефектов контраст в ИК-диапазоне оставался существенным и легко измеряемым и при значительной роли теплопроводности, что позволило обнаруживать и характеризовать дефекты вплоть до субмиллиметрового масштаба. Важно отметить, что законы концентрации электрического тока и механических напряжений

$5^{*}$ Письма в ЖТФ, 2017, том 43, вып. 19 


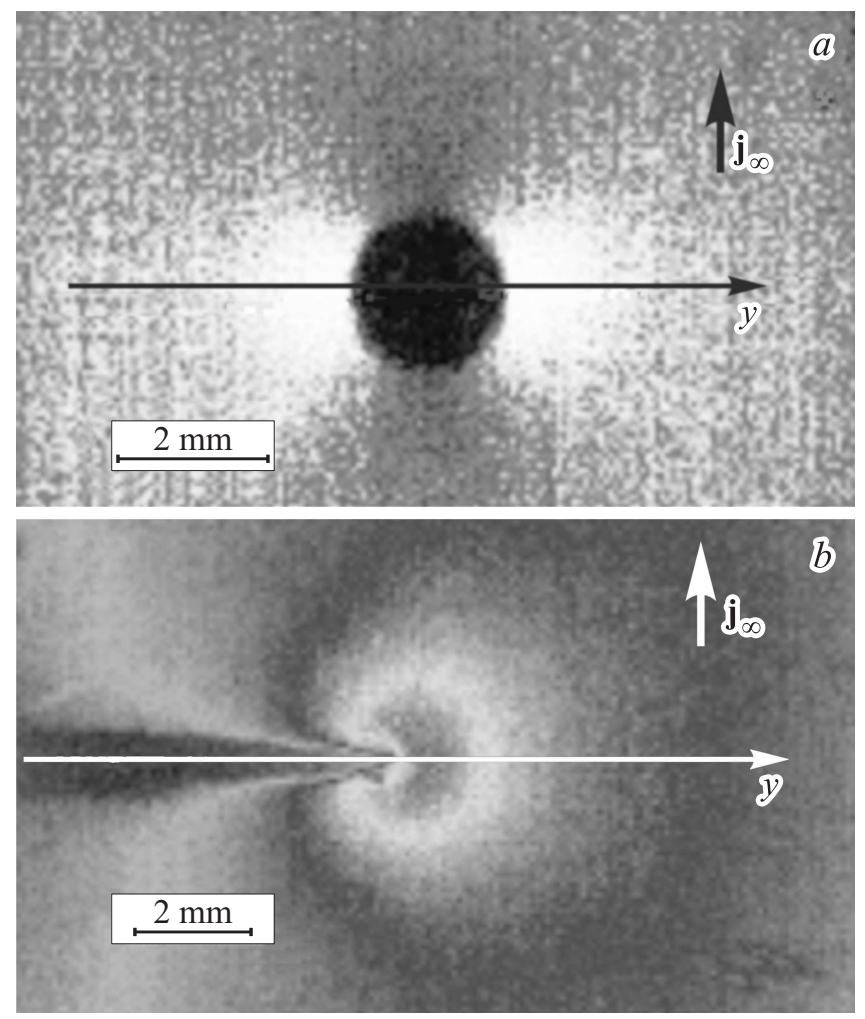

Рис. 1. Разность тепловых полей непосредственно до и после прохождения прямоугольного импульса 150 А длительностью $117 \mathrm{~ms}$ по стальной пластине с круглым отверстием диаметром $2 \mathrm{~mm}(a)$ и прямоугольного импульса $30 \mathrm{~A}$ длительностью $733 \mathrm{~ms}$ по стальной пластине с боковой перпендикулярной трещиной длиной $6.4 \mathrm{~mm}(b)$.

на несплошностях любой геометрии подобны. По этой причине градиенты температурного поля и контраст тепловизионного изображения тем выше, чем более опасны дефекты с точки зрения вероятности зарождения и последующего распространения трещин.

Таким образом, в работе показано, что электротоковая термометрия при последующей обработке тепловизионного изображения, регистри-

Письма в ЖТФ, 2017, том 43, вып. 19 

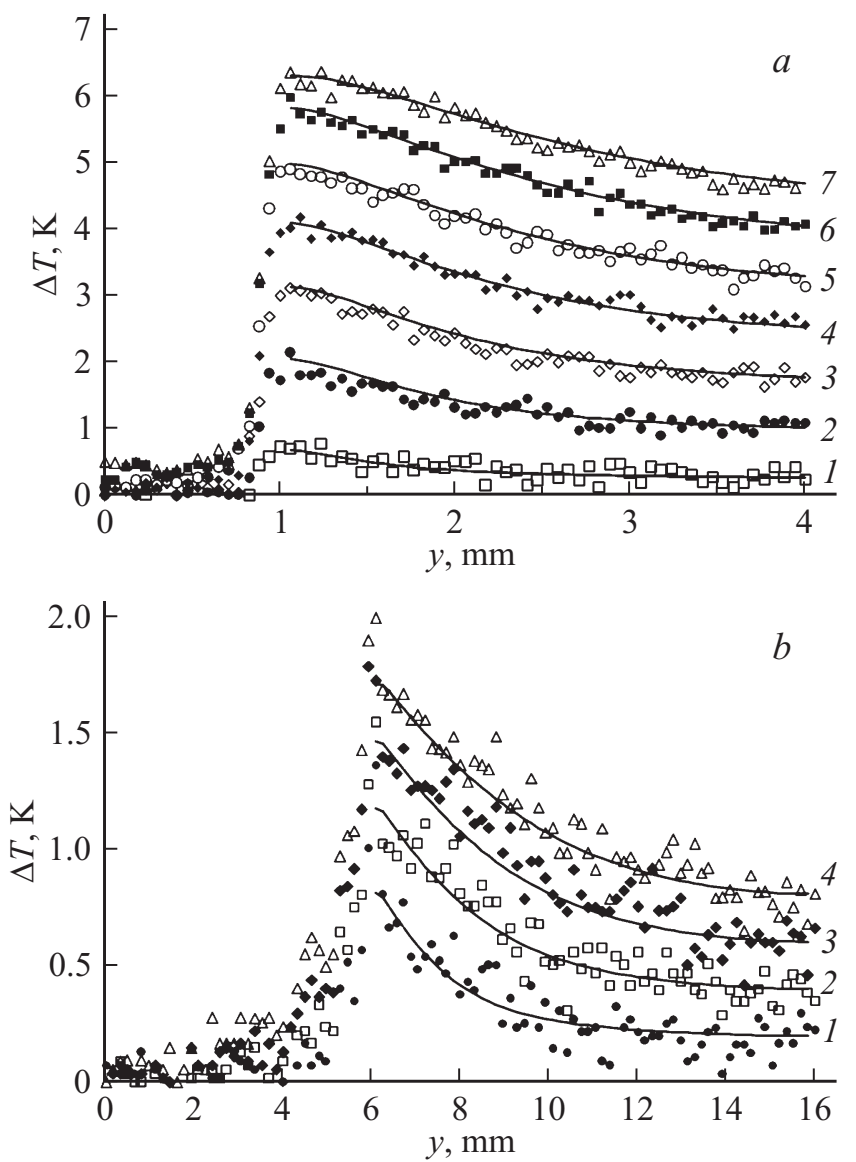

Рис. 2. Разности температурных профилей полей. $a-$ непосредственно до и в процессе прохождения прямоугольного импульса тока 150 А длительностью $117 \mathrm{~ms}$ по стальной пластине с круглым отверстием диаметром $2 \mathrm{~mm}$ вдоль оси $y$ в разные моменты времени, ms: $1-16.7,2-33.3,3-50,4-$ $66.7,5-83.3,6-100,7-116.7 . b-$ непосредственно до и в процессе прохождения прямоугольного импульса тока $30 \mathrm{~A}$ длительностью $733 \mathrm{~ms}$ по стальной пластине с боковой перпендикулярной трещиной длиной $6.4 \mathrm{~mm}$ вдоль оси $y$ в разные моменты времени, ms: $1-183,2-366,3-549,4-733$. Время отсчитывалось от момента начала пропускания тока по пластине. Экспериментальные данные показаны точками, результаты моделирования сплошными линиями.

Письма в ЖТФ, 2017, том 43, вып. 19 
руемого даже с не очень высокой скоростью съемки (время между кадрами больше характерного времени термической диффузии), позволяет корректно характеризовать геометрические размеры обнаруживаемых дефектов с субмиллиметровой точностью и определять коэффициент температуропроводности материала с точностью $\sim 10 \%$ или лучше. При этом можно использовать дешевые и портативные источники тока небольшой импульсной мощности и тепловизоры с невысокой скоростью сьемки, позволяющие разработать легкий переносной прибор для контроля котлов, трубопроводов, емкостей в производственных или полевых условиях.

Разработка новой комбинированной методики и портативного прибора для оперативной диагностики дефектов, а также проведение экспериментальных исследований выполнены при поддержке гранта Российского научного фонда (проект № 15-19-00181). Разработка аналитических и численных моделей осуществлена при поддержке гранта РФФИ (проект № 17-48-680817).

\section{Список литературы}

[1] Ibarra-Castanedo C., Maldague X.P. // Handbook of technical diagnostics: fundamentals and application to structures and systems / Ed. H. Czichos. Berlin: Springer-Verlag, 2013. P. 175-220.

[2] Вавилов В.П. Инфракрасная термография и тепловой контроль. М: Спектр, 2009. $544 \mathrm{c}$.

[3] Oswald-Tranta B. // Nondestruct. Test. Eval. 2007. V. 22. P. 137-153.

[4] Usamentiaga R., Venegas P., Guerediaga J., Vega L., Molleda J., Bulnes F.G. // Sensors. 2014. V. 14. P. 12305-12348.

[5] Vavilov V.P., Burleigh D.D. // NDT \& E International. 2015. V. 73. P. 28-52.

[6] Balageas D., Maldague X., Burleigh D., Vavilov V.P., Oswald-Tranta B., Roche J.M., Pradere C., Carlomagno G.M. // J. Nondestruct Eval. 2016. V. 35. P. 18 (1-17).

[7] Libin M.N., Balasubramaniam K., Maxfield B.W., Krishnamurthy C.V. // AIP Conf. Proc. 2013. V. 1511. P. 539-546.

[8] Gao B., Woo W.L., Tian G.Y. // Sci. Rep. 2016. V. 6. P. 25480 (1-12).

[9] Yang R., He Y. // Appl. Phys. Lett. 2015. V. 106. P. 234103 (1-4)

[10] Головин Ю.И. Наноиндентирование и его возможности. М.: Машиностроение, 2009. 312 c.

[11] Сидоров Ю.В., Федорюк М.В., Шабунин М.И. Лекции по теории функций комплексного переменного. М.: Наука, 1989. 480 с.

Письма в ЖТФ, 2017, том 43, вып. 19 